Case Report

\title{
CT Scan Features of Presumptive Haemorrhagic Stroke in a Dog with Cushing's Disease
}

\author{
A. Liotta, ${ }^{1}$ R. Cavrenne, ${ }^{1}$ D. Peeters, ${ }^{2}$ J. Manens, $^{2}$ and G. Bolen ${ }^{1}$ \\ ${ }^{1}$ Diagnostic Imaging Section, Department of Small Animals and Equidae Clinic, Faculty of Veterinary Medicine, University of Liege, \\ Boulevard de Colonster 20, 4000 Liege, Belgium \\ ${ }^{2}$ Internal Medicine Section, Department of Small Animals and Equidae Clinic, Faculty of Veterinary Medicine, University of Liege \\ Boulevard de Colonster 20, 4000 Liege, Belgium \\ Correspondence should be addressed to A. Liotta; apliotta@ulg.ac.be
}

Received 12 April 2014; Accepted 23 June 2014; Published 3 July 2014

Academic Editor: Silke Hecht

Copyright @ 2014 A. Liotta et al. This is an open access article distributed under the Creative Commons Attribution License, which permits unrestricted use, distribution, and reproduction in any medium, provided the original work is properly cited.

\begin{abstract}
A 9-year-old, intact male, Brie's shepherd dog, with a 10-day history of depression and tachypnoea developed signs of central neurological dysfunction. 16 Multislice Computed Tomography (CT) pre- and postcontrast studies of the brain revealed a single intra-axial homogeneous well-circumscribed hyperattenuating $(+/-62 \mathrm{HU})$ and noncontrast-enhancing area, $5 \mathrm{~mm}$ in diameter, in the caudal part of the mesencephalon. This finding was highly suggestive of a haemorrhagic event. A pituitary-dependent hyperadrenocorticism (PDH) was identified and is considered likely to be the underlying cause. A repeat CT scan examination, 2 months later, showed almost complete resolution of the brain lesion. The present case describes a solitary 5 mm diameter lesion: the result of intracranial haemorrhage in a dog with presumed PDH.
\end{abstract}

\section{Case Presentation}

A 9-year-old, entire male, Brie's shepherd dog, was referred with a 10-day history of acute depression and tachypnoea. On clinical evaluation, the only abnormalities detected were obtundation and superficial tachypnoea. A complete blood count, biochemistry profile, blood gas analysis, and urinalysis revealed only a mild increase in serum GGT, ALT, and cholesterol. Systolic blood pressure (Doppler method) was within normal limits. Thoracic radiographs were unremarkable. Three days later, the dog developed acute neurological signs consistent with central dysfunction with right-sided amaurosis, ventromedial strabismus of the left eye, and leftsided Horner's syndrome.

16 Multislice Computed Tomography pre- and postcontrast studies of the brain were performed. A single intraaxial, homogeneous, well-circumscribed, irregularly shaped, hyperattenuating (+/- $62 \mathrm{HU})$, and noncontrast-enhancing area of $5 \mathrm{~mm}$ diameter was observed in the caudal part of the mesencephalon, mildly left-sided, exerting a mild mass effect on the surrounding structures and associated with dilation of the ventricular system (Figure 1). This finding was highly suggestive of a haemorrhagic event. Potential underlying causes included hypertension, thromboembolism or coagulopathy, or haemorrhagic tumours (haemangioma, haemangiosarcoma, and haemorrhagic gliomas). Cerebrospinal fluid collection was performed; protein levels and cell count were within normal limits. A coagulation profile (prothrombin time, activated partial thromboplastin time, and thrombin time) was normal. Abdominal ultrasound showed a bilateral severe, well marginated, homogeneous enlargement of right and left adrenal glands measuring, respectively, $1,5 \mathrm{~cm}$ and $2 \mathrm{~cm}$. PDH may therefore be suspected. Less likely, the increase in size of the adrenal glands may occur secondary to bilateral adrenal neoplasia, such as functional or nonfunctional carcinoma and adenoma, phaeochromocytoma, granuloma, or myelolipoma. The neurologic abnormalities improved gradually until complete resolution 30 days after the initial clinical presentation. At that time, a low-dose dexamethasone suppression test was performed and confirmed the diagnosis of PDH (in both samples 4 and 8 hours after the injection, the value was above the normal limits of $1 \mu \mathrm{g}$, 


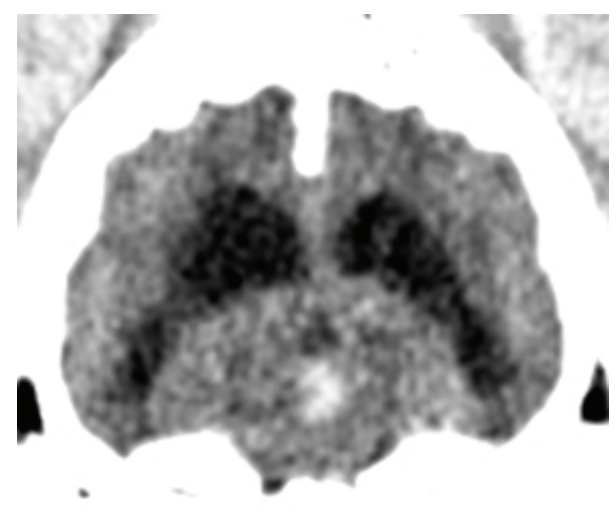

FIgURE 1: Transverse CT image of the brain prior to contrast medium administration showing a small intra-axial hyperattenuating lesion in the mesencephalon and a moderate asymmetric enlargement of the ventricular system.

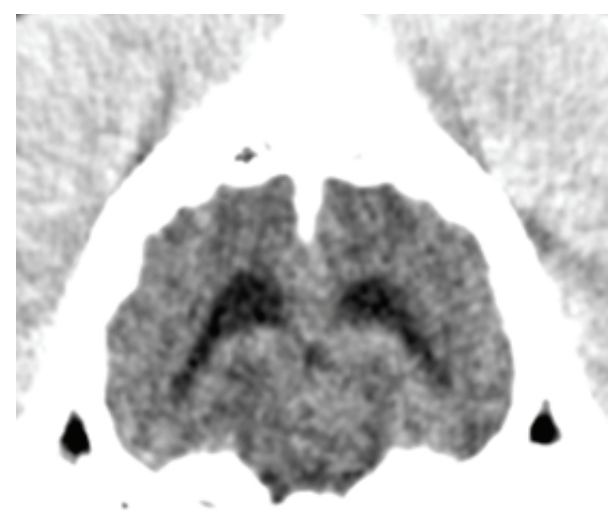

FIgURE 2: Transverse CT image of the brain, 30 days later, showing the absence of the lesion and the almost complete resolution of the ventricular enlargement.

respectively, 1,68 and $3,06 \mu \mathrm{g}$ with a $>50 \%$ reduction in the 4-hour sample compared to the basal cortisol value). The CT scan was repeated 2 months later to follow the evolution of the lesion and showed almost complete resolution of the brain lesion, including resolution of the ventricular system enlargement (Figure 2). The improvement in the CT scan images corresponded with a complete resolution of clinical signs. In both CT examinations, the pituitary gland appeared within normal limits measuring $6 \times 4 \times 4 \mathrm{~mm}$ with a pituitary gland/brain area ratio of $<0,31$.

Two months after initial presentation the patient was lost to follow-up.

\section{Discussion}

Diagnosis of intracranial haemorrhage is based on CT and MRI: the former is very sensitive and was previously the primary diagnostic tool for detection of haemorrhage in human medicine. MRI provides a more accurate estimate of the stage of haemorrhage by identifying sequential patterns of degradation of the haemoglobin molecule during haematoma transformation [1].

There is a linear relationship between CT attenuation, protein content (mainly haemoglobin), and haematocrit. In the early hours of haemorrhage, the lesion exhibits moderate high-density values, because of freshly extravasated blood characterized by cells interspersed with protein-rich serum. The increase of haematocrit in the subsequent period gives the lesion the aspect of a well-demarcated hyperattenuated lesion, with Hounsfield units' values reaching 60-80 HU. With clot retraction the CT attenuation values increase to $80-100 \mathrm{HU}$ in the centre of the haematoma, while a hypoattenuating halo surrounds the central nidus due to serum extrusion and reactive vasogenic oedema. In the weeks following the acute event the attenuation of the haematoma decreases as proteolysis begins, even if the mass effect persists. Within weeks or months, the digestion of blood degradation products by macrophages can lead to the total resolution of the lesion, even if calcification, hypoattenuating area, focal atrophy, or ventricular enlargement can be easily detected on CT images and can represent the sequel of an old haematoma [1].

Primary intracranial haemorrhage is less frequent than secondary intracranial haemorrhage and is considered very rare in animals [2]. Some neoplastic conditions may lead to secondary intracranial haemorrhage because of concurrent disseminated intravascular coagulopathy or because of bleeding. Examples of such tumours include haemangiosarcoma [3-5], haemangioma [6], oligodendrogliomas, glioblastomas, ependymomas, or pituitary adenomas [4]. Many different pathological conditions have also been associated with secondary intracranial bleeding: trauma [7], congenital vascular malformations [8, 9], vasculitis [2], Angiostrongylus vasorum infection [10,11], and systemic arterial hypertension [10].

The diagnosis of hyperadrenocorticism (HAC) is challenging and none of the biochemical screening or differentiating tests is perfect $[12,13]$. The low-dose dexamethasone suppression test is considered the test of choice, unless iatrogenic HAC is suspected. The sensitivity of this test is excellent ranging from 85 to $100 \%$, but specificity is lower ranging from 44 to $73 \%$, especially when measured in a population of sick dogs, affected by chronic disease $[12,13]$. Because of the influence of possible concurrent nonadrenal disease the dog described here was tested after a delay of 30 days, when it had recovered and was not showing clinical signs. The dog in this study showed bilateral increase in the size of the adrenal glands. This finding is consistent with the diagnosis of HAC. Other differential diagnoses such as bilateral nonfunctional adrenal neoplasia, granuloma, or myelolipoma seem less likely, but could not be completely ruled out.

In the present case, the pituitary gland appeared within normal limits on CT examination, but one limitation is that a dynamic CT examination was not performed. However, even if dynamic CT provides very useful information in additional to conventional contrast-enhanced CT, the absence of abnormalities on dynamic CT examination does not rule out the presence of a microadenoma [14]. 
The incidence of HAC in dogs with intracranial haemorrhage is at present unknown. In a study of Garosi et al. [15], HAC was cited as a potential underlying cause of cerebrovascular accident in dogs, but all reported cases involved ischaemic infarction. In another study [16], one of 4 dogs with haemorrhagic stroke had been under treatment for HAC for 8 months. The presence of a hypercoagulable state in conjunction with mild to moderate systemic hypertension leading to vascular lesions represents a possible explanation for haemorrhagic stroke in dogs with HAC $[4,10]$. Recently, a study described concurrent medical conditions and longterm outcomes in dogs with nontraumatic intracranial haemorrhage. In that study, 5 of 75 dogs had systemic hypertension and $2 / 5$ were diagnosed with HAC. In all 5 cases, MRI examination showed multiple lesions less than $5 \mathrm{~mm}$ in size [10]. This is similar to what happens in people, where systemic hypertension is commonly seen in association with microbleeding, that is, multiple haemorrhages $<5 \mathrm{~mm}$. The dog in this case differed from what is typically described in the literature because of the absence of hypertension detected during the clinical examination and the presence of a solitary lesion of $5 \mathrm{~mm}$ in diameter. Therefore, it is reasonable to postulate that in our case the relationship between HAC and the intracranial lesion may not be related to a hypertensive event; the pathogenesis remains unknown.

The absence of histological confirmation for both the suspicion of intracranial haemorrhage and the pituitarydependent HAC represents a limitation in this case. However, the resolution of the lesion on the second CT examination and the absence of clinical signs two months after the event support our suspicion of intracranial haemorrhage. On the other hand given the positive low-dose dexamethasone suppression test and the bilateral increase in adrenal gland size we can postulate that pituitary-dependent HAC is the most likely underlying disease, although this diagnosis could not be confirmed.

In conclusion, the present case describes an intracranial haemorrhage in a dog with suspected Cushing's disease as a single lesion of $5 \mathrm{~mm}$ diameter.

\section{Conflict of Interests}

The authors declare that there is no conflict of interests regarding the publication of this paper.

\section{Acknowledgment}

The authors would like to thank Dr. F. Allerton for English grammar editing.

\section{References}

[1] P. M. Parizel, S. Makkat, E. Van Miert, J. W. Van Goethem, L. Van den Hauwe, and A. M. De Schepper, "Intracranial hemorrhage: principles of CT and MRI interpretation," European Radiology, vol. 11, no. 9, pp. 1770-1783, 2001.

[2] L. S. Garosi, "Cerebrovascular disease in dogs and cats," Veterinary Clinics of North America: Small Animal Practice, vol. 40, no. 1, pp. 65-79, 2010.
[3] D. J. Waters, D. W. Hayden, and P. A. Walter, "Intracranial lesions in dogs with hemangiosarcoma," Journal of Veterinary Internal Medicine, vol. 3, no. 4, pp. 222-230, 1989.

[4] A. Wessmann, K. Chandler, and L. Garosi, "Ischaemic and haemorrhagic stroke in the dog," Veterinary Journal, vol. 180, no. 3, pp. 290-303, 2009.

[5] M. Dennler, E. Lange, O. Schmied, and B. Kaser-Hotz, "Imaging diagnosis-metastatic hemangiosarcoma causing cerebral hemorrhage in a dog," Veterinary Radiology and Ultrasound, vol. 48, no. 2, pp. 138-140, 2007.

[6] B. M. Eichelberger, S. L. Kraft, C. H. C. Halsey, R. D. Park, M. D. Miller, and L. Klopp, "Imaging diagnosis-magnetic resonance imaging findings of primary cerebral hemangioma," Veterinary Radiology \& Ultrasound, vol. 52, no. 2, pp. 188-191, 2011.

[7] M. Kitagawa, M. Okada, K. Kanayama, and T. Sakai, “Traumatic intracerebral hematoma in a dog: MR images and clinical findings," Journal of Veterinary Medical Science, vol. 67, no. 8, pp. 843-846, 2005.

[8] W. R. Hause, M. L. Helphrey, R. W. Green, and P. C. Stromberg, "Cerebral arteriovenous malformation in a dog," Journal of the American Animal Hospital Association, vol. 18, pp. 601-607, 1982.

[9] W. B. Thomas, W. H. Adams, M. D. Mcgavin, and R. E. Gompf, "Magnetic resonance imaging appearance of intracranial hemorrhage secondary to cerebral vascular malformation in a dog," Veterinary Radiology and Ultrasound, vol. 38, no. 5, pp. 371-375, 1997.

[10] M. Lowrie, L. de Risio, R. Dennis, F. Llabrés-Díaz, and L. Garosi, "Concurrent medical conditions and long-term outcome in dogs with nontraumatic intracranial hemorrhage," Veterinary Radiology and Ultrasound, vol. 53, no. 4, pp. 381-388, 2012.

[11] M. Zarelli, R. Shiel, B. Gallagher, C. Skelly, S. Cahalan, and H. Mcallister, "Imaging diagnosis: Ct findings in a dog with intracranial hemorrhage secondary to angiostrongylosis," Veterinary Radiology and Ultrasound, vol. 53, no. 4, pp. 420-423, 2012.

[12] E. N. Behrend, H. S. Kooistra, R. Nelson, C. E. Reusch, and J. C. Scott-Moncrieff, "Diagnosis of spontaneous canine hyperadrenocorticism: 2012 ACVIM consensus statement (small animal)," Journal of Veterinary Internal Medicine, vol. 27, no. 6, pp. 1292-1304, 2013.

[13] M. E. Peterson, "Diagnosis of hyperadrenocorticism in dogs," Clinical Techniques in Small Animal Practice, vol. 22, no. 1, pp. 2-11, 2007.

[14] R. H. van Der, B. P. Vlugt-Meijer, T. S. Van den Ingh, A. Rijnberk, and G. Voorhout, "Dynamic computed tomography of the pituitary gland in dogs with pituitary-dependent hyperadrenoc orticism," Journal of Veterinary Internal Medicine, vol. 17, pp. 773-780, 2003.

[15] L. Garosi, J. F. McConnell, S. R. Platt et al., "Results of diagnostic investigations and long-term outcome of 33 dogs with brain infarction (2000-2004)," Journal of Veterinary Internal Medicine, vol. 19, no. 5, pp. 725-731, 2005.

[16] A. E. H. Paul, Z. Lenard, and C. S. Mansfield, "Computed tomography diagnosis of eight dogs with brain infarction," Australian Veterinary Journal, vol. 88, no. 10, pp. 374-380, 2010. 

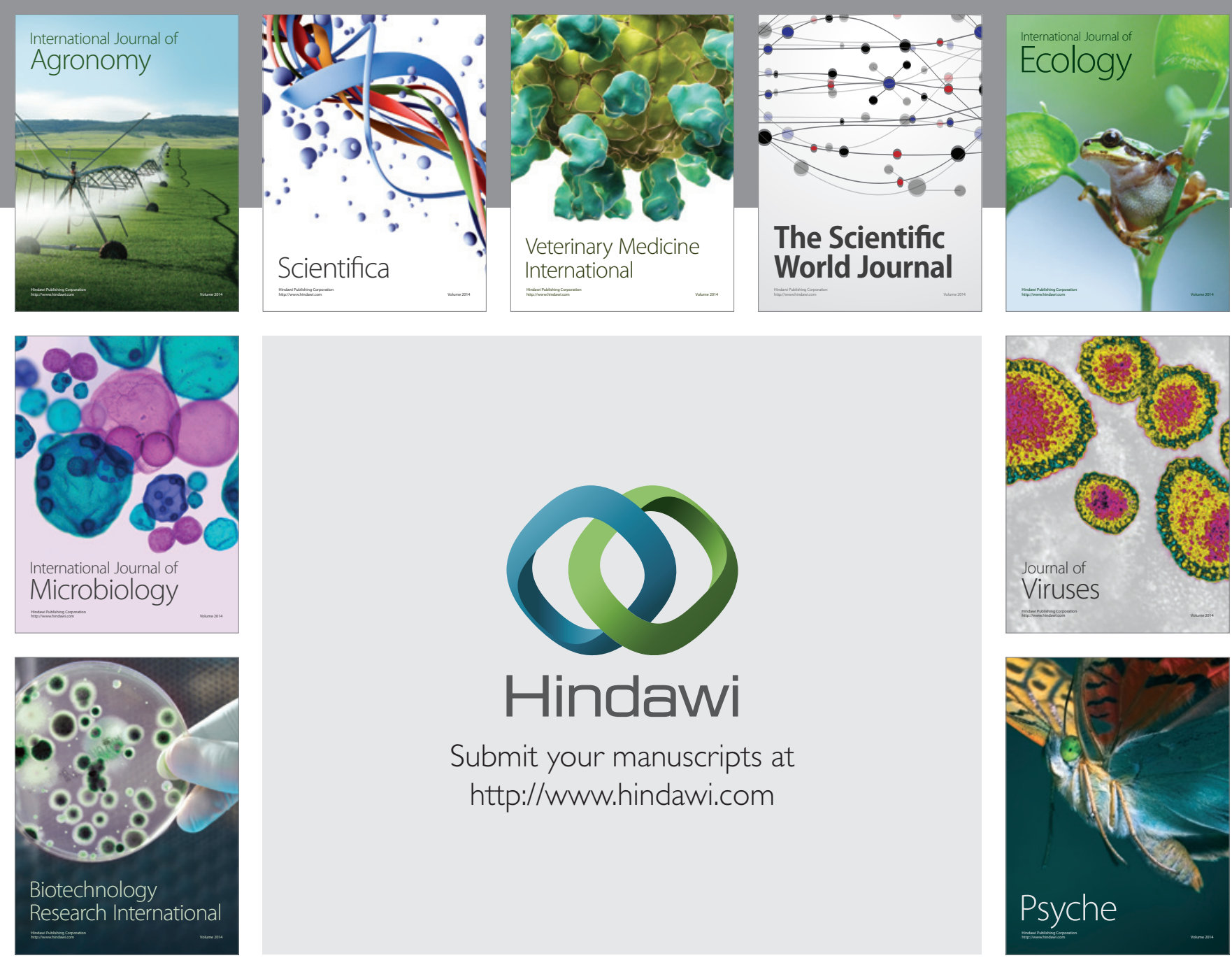

Submit your manuscripts at http://www.hindawi.com
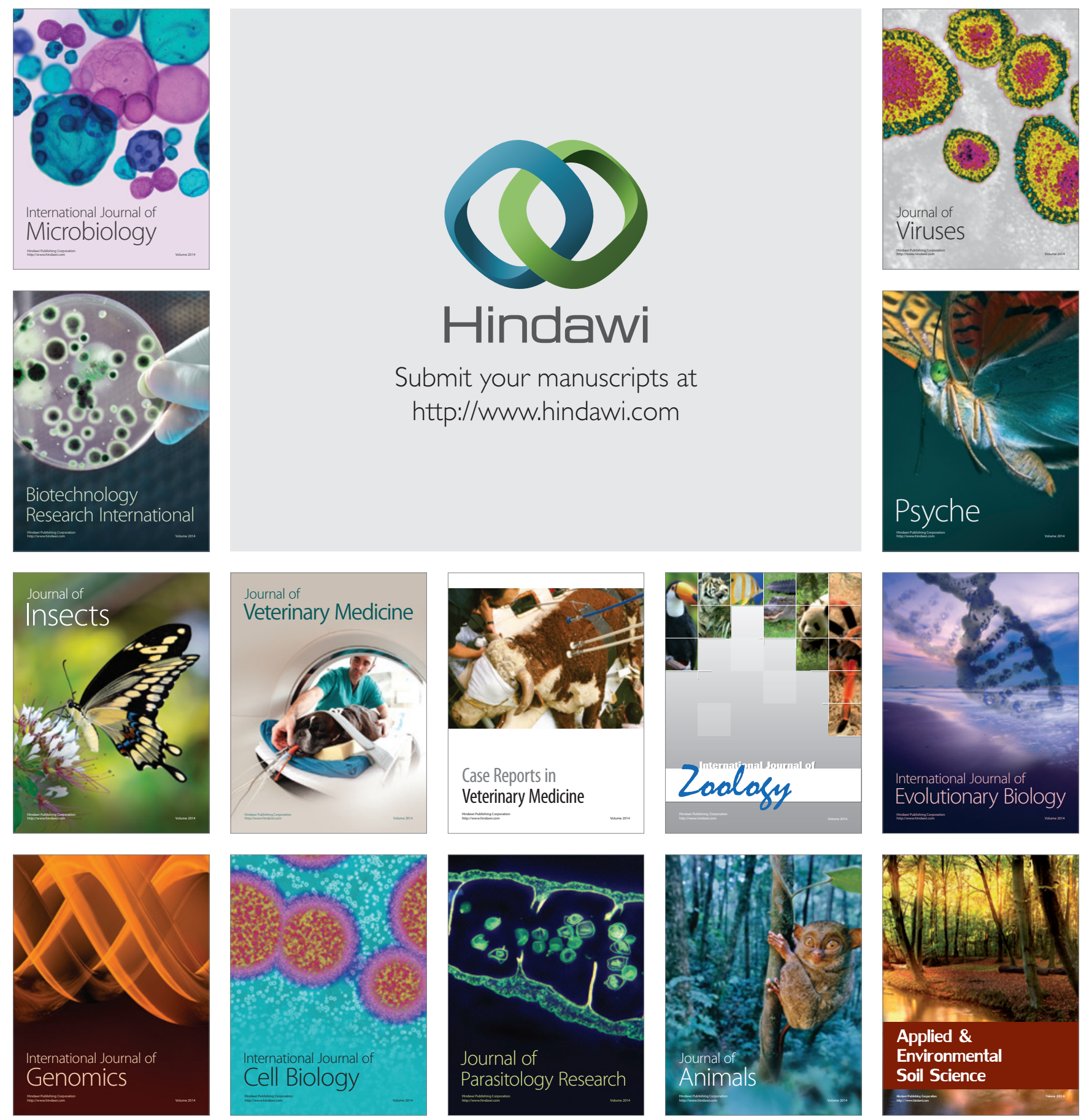